\title{
CIENCIA Y GÉNERO: AUTORIDAD Y MEDIDA EN LA ENSEÑANZA*
}

\author{
SUSI, E. \\ Instituto LAMEL-CNR. Via Gobetti, 101. Bologna. Italia.
}

\section{SUMMARY}

The report begins with the division which exists between teaching and research, which is considered to be a result of science's incapacity for speaking about itself and transmitting the sense of knowledge which it develops. Attention is drawn to the negligible relationship between those who carry out research and those who teach and the lack of authority which this causes. It is the root of the problem which many teachers encounter in establishing proper limits for themselves and for their own teaching of disciplinary knowledge. As a result of this lack of mediation, there is on the one hand an osciliation between biind subjection and impotent criticism which characterizes the relation of those who are not scientifically qualified, and the other hand the dual excess of arrogance and silence on the part of those who are qualified and who thus severely limit communication between science and society.

In this situation, one sees a condition shared by many women with regard to work, politics and science. It is in the political practice of female relationships and in the theoretical elaboration of how authority is formed and evolves that the place where appropriate questions have found order and meaning is revealed.

From information regarding the need for mediation efforts to encourage communication between teaching and research, the report goes on to evaluate those which already exist, discussing the conditions under which a science of education might unite the nurturing of such a relation with the objectivity which is necessary.

\section{CUANDO LA COMPETENCIA NOS ENMUDECE: LA SEPARACION ENTRE ENSENANZA E INVESTIGACIÓN}

Cuando recibí la invitación para intervenir en esta conferencia, me quedé algo perpleja. Me preguntaba qué podría decir a una convención de enseñantes que les interesara, yo que no he enseñado nunca.

Os hablo de mi reticencia inicial porque me parece que es un signo de cómo ha llegado a considerarse normal la idea de que la enseñanza y la práctica de la ciencia son dos cosas separadas. No siempre ha sido así, más bien se puede decir que esta separación es una característica de nuestros días. No es necesario retroceder mucho en la historia de la ciencia, para encontrar grandes escuelas cientifficas que se formaban en la universidad y en las cuales, las clases eran una fase de la investigación. Por ejemplo:

Muchas innovaciones matemáticas fundamentales se comunicaron directamente en las clases, en sus textos y apuntes. El gran matcmático alemán, Weierstrass, por citar a uno de tantos, que vivió entre 1815 y 1897 , fue durante mucho tiempo profesor de instituto $y$, precisa- mente, en este período profunđizó en las sistematización rigurosa del análisis, a la que está ligado su nombre, y que le supuso la llamada para enseñar en la universidad. Una parte fundamental đe su trabajo se conoció a través de sus clases en Ia Universidad de Berlín, en las que, entre otros, se formó Georg Cantor. Peano dedicaba muchas horas ć. la didáctica de las matemáticas en la escuela elemental.

Ahora, la enseñanza, no sólo la de los primeros niveles, sino támbién la universitaria, se considera por muchas personas como una rutina aburrida que resta un tiempo precioso a la investigación.

Las causas de la separación entre el «hacer»y el «enseñar ciencisı"

Las razones de es 'e estado de cosas son muchas y vale la pena pararse un momento, porque dejan claros algunos aspectos importantes del modo actual de hacer ciencia: 
a) La especialización creciente de la competencia disciplinar específica.

b) Un conocimiento científico muy separado de la experiencia personal: lenguaje específico.

c) Una valoración social diferente.

d) La dificultad para transmitir el sentido del hacer científico.

a) Ante todo cuenta la creciente separación de las materias, necesaria por la vastedad del saber acumulado, por la cual se segmentan las actividades intelectuales en especialidades cada vez más restringidas e incomunicadas entre sí. Esta división hace que ganemos en profundidad y precisión, pero al mismo tiempo es causa de una perdida de visión general y de capacidad de síntesis e intercambio.

b) Una consecuencia es que la experiencia personal y el conocimiento del mundo quedan lejanos del conocimiento científico. Se tiende a elaborar un lenguaje específico y críptico.

¿Cómo enseñar dentro de una concepción, no sólo de transmisión de conocimientos sino en el sentido de una actividad cognoscitiva dentro de un punto de vista general, si es necesario juntando fatigosamente pedacitos de saber proporcionados por las personas especialistas?

c) La enseñanza, cuando no concierne a la estricta competencia disciplinar, cuando, por lo tanto, sale del ámbito reproductivo de la comunidad científica de especialistas, necesita transmitir el sentido de la actividad cognoscitiva y, consecuentemente, disponer de un punto de vista más general. Para ello es necesario un empeño y un esfuerzo cada vez más voluntarioso, ya que no tiene recompensas en forma de prestigio social o de promoción en la carrera docente.

Un segundo elemento que apoya la división entre enseñanza e investigación es la polarización de la atención social sobre las actividades que tienen una repercusión inmediata en la economía y la menor atención que, siempre, dedican las sociedades económicamente desarrolladas a la transmisión del saber, cuya importancia en ei desarrollo económico es demasiado indirecta y dilatada en el tiempo. De ello se deriva que los recursos dedicados a la enseñanza son insuficientes y que su prestigio social es escaso.

Por el contrario, la importancia económica de la investigación científica produce una presión social sobre las personas que se dedican a esa actividad en el sentido de incitarlas a una productividad cada vez mayor, a través de mecanismos de financiación y de la estructura de la carrera profesional, que desvaloriza todas aquellas actividades que no vayan a obtener resultados inmediatos, susceptibles de utilización.

d) La dificultad para transmitir el sentido del hacer científico repercute en que una gran parte de la investigación avanzada carece de un terreno de confrontación y mediacion con una parte importante de la cultura y de las necesidades y expectativas de la sociedad de la que forma parte. Esto trae consigo diversas consecuencias:

- Para quienes se dedican a la investigación científica.

- Para quienes se dedican a la enseñanza.

- Para aquelliss personas que se dedican a la investigación científica:

- Afasia de la ciencia.

- Falta de mejiación entre la ciencia y la sociedad.

- Arrogancia $\supset$ silencio.

De hecho, la tendencia natural de quienes se dedican a la investigación es la de dirigirse exciusivamente a sus comunidades disciplinares, en las cuales su competencia puede circular y ser reconocida sin posteriores mediaciones, limitardo su relación directa con la sociedad, si es que se da, a' plano de la divulgación. Pasa a segundo plano la verdád de la ciencia, sustituyéndose por el reconocimientio de la comunidad cientifica. No hay disposición a escuchar las opiniones de otros.

Dependiendo del gusto o de la vanidad personal, habrá quien se limitará a manifestarse sólo sobre lo que concieme estrictamente a su campo de conocimiento y quien, por el contrario, se dejará tentar por divulgar argumentos diversos. Entonces, el lenguaje de la ciencia acaba oscilands entre el refinamiento críptico de la especialización y la simplificación brutal de la divulgación-espectáculo, practicada por la televisión y la prensa.

La ciencia pareze incapaz de hablar de sus resultados, que transforman la realidad tan profundamente, con un lenguaje transparente, comprensible para una mayoría, sin traicionar el rigor de los experimentos de los que nace.

- Para quienes se dedican a la enseñanza, las consecuencias son otras:

- Falta de acceso al lugar donde se produce el saber científico.

- Incertidumbre y dificultad para acceder a una expresión coherente con la propia experiencia del mundo.

- Problemas de autoridad.

La existencia de una amplio grupo de enseñantes que no tiene acceso directo a los lugares en los que se produce el saber científico, ni dispone de instrumentos propios de mediación con estos últimos, produce también una afasia similar.

En efecto, sobre quien enseña, se producen exigencias contradictorias. Por un lado, la transmisión del saber demanda una asunción de autoridad, ya sea en la relación con el alumnado o en su propio enfrentarse a la materia, por la necesidad de seleccionar contenidos y de decidir 
la profundidad con que se han de tratar. No olvidemos que la ciencia liega construida a la escuela.

Por otro lado, Ia ausencia de relaciones con la comunidad científica, en la que se construye y se hace circular Ia autoridad de la ciencia, hace vivir como arbitraria y no suficientemente reconocida esta asunción de autoridad, sensación reforzada por la escasa consideración social y la dificultad objetiva de alcanzar el nivel justo de competencia sobre un número creciente de temas, cada uno de los cuales es rico en novedades complejas.

Se da entonces aquello que Angela Alioli (1992), en su texto, «Una matemática que enseña», llama «el problema infinito", es decir, el no saber poner término a la búsqueda de la competencia. Dice Alioli:

«Las respuestas a las preguntas de qué eran las matemáticas y con qué objetivo se enseñaban, que me comunicaba la comunidad de expertos en la que estaba inserta, eran extrañas a mis expectativas, no bastaban para dejarme entrat tranquila en clase.

»Alejada de otra comunidad a la que dirigirme, decidí ponerme al día. Aprendía cosas nuevas, aburridas o interesantes, me apasionaba, acumulaba material sobre aproximaciones didácticas diversas, pero lo que no sabía parecía no disminuir jamás.»

Un gran número de enseñantes que tiene gran deseo de calidad en su trabajo conoce la misma angustia: sigue infinitos cursos de actualización, busca con atención las últimas novedades en las revistas especializadas, ajusta continuamente el programa. También está, por oposición, quien durante años enseña «por sus apuntes», ignorando todo lo que ha cambiado. En el fondo, se trata de manifestaciones diversas de la dificultad de encontrar una medida adecuada de la propia persona y de su proyecto didáctico, que produce y transmite la misma dificultad para acceder a un discurso coherente con el propio estar en el mundo.

No hay un único método para enseñar matemáticas, cada enseñante debe desarrollar el suyo, pero para ello es imprescindible la autoridad.

\section{CUESTIÓN DE AUTORIDAD}

La autoriđad científica:

- Nace de las relaciones que constituyen la comunidad científica.

- Tiene una impronta masculina muy fuerte.

- Oculta esta particularidad bajo el supuesto de la neutralidad científica. Ignora la relación entre el sujeto y el objeto.

En el caso de la investigación, el problema no está en la separación de la comunidad científica, sino, por así decirlo, en un exceso de pertenencia que impide ver lo que sucede; en particular, impide ver la relación entre pertenecer a la comunidad y construir autoridad.

De hecho, muchas científicas y científicos consideran como algo issencial de su propio trabajo la relación con el objeto d $\epsilon$ estudio, la verdad a descubrir, las hipótesis a probar, y tienen el convencimiento de que sólo en su capacidad para capturar la verdad contenida en las matemáticas, la física, etc. está la fuente de la credibilidad de sus resılitados $\mathrm{y}$, por tanto, la autorización para venderlos socialmente. Pasa entonces a segundo plano, el elemento esencial de la empresa científica, es decir, $e l$ hecho de que la verdad cientifica se forma y se reconoce a través de relaciones entre cientificos y que los resultados obteridos por grupos de investigación o por investigadores solitarios sólo son examinados por la comunidad científica, para obtener el reconocimiento de validez que permita presentarlos públicamente.

En sintesis, la autoridad de la ciencia no es el fruto de abstractas cualidades del método científico, sino de concretas rolaciones sociales; es decir, que la ciencia no dice una verdad abstracta y absoluta, pero dice «lo verdadero" a través de las relaciones, y es en ellas donde se forma y sircula entre los investigadores, la autoridad para decir la verdad.

Esta validación del trabajo por parte de la comunidad cientifica no existe para quienes se dedican a la enseñanza

Si se pierde de yista este hecho decisivo, se acaba por pensar que tenemos autorización para expresar nuestras propias opiniones, porque estamos en posesión de una competencia especializada que sostiene este derecho por sí misma. Nos volvemos arrogantes, sin disposición para escuchar preguntas u otras opiniones, o bien conscientes de la pequeñez del trocito de verdad que poseemos; tenemos afiasia para todo to que no afecte estrictamente a la propia competencia específica, que para una persona muy escrupiulosa, puede querer decir casi todo.

\section{La situacioin de las mujeres}

Hablar requiere autoridad simbólica. Muchas mujeres han vivido $y$ viven en condiciones de afasia en el trabajo, en la política, en la ciencia y ha sido justamente en la práctica política y en el trabajo de algunas mujeres donde he encontrado la clave para entender las razones y observar las posibilidades de cambio que la realidad ofrece. Estia clave es la autoridad.

Luisa Murcno (1991) en su libro El orden simbólico de la madre, hablando de «la dificultad femenina histórica mente determinada a manifestar su propia experiencia», afirma que «[...] se trata del problema planteado de la falta de autoridad simbólica, es decir, la autoridad de afirmar aquello que es, que la lengua posee y que hacemos nuestra aprendiendo a hablar. En ausencia de esta autoridad, no se pierde necesariamente la competenica linguística, lacual permanece para cubrirel defecto de otra competencia más elemejtal que llamaré simbólica y que consiste en tener senticlo de la mediación y de su necesidad [...] 
"La incompetencia simbólica, de hecho, trasciende el nivel lingüístico, haciendo nacer en la persona que habla la incertidumbre de que las palabras puedan decir aquello que queremos decir, y empujándola a buscar recursos para vencer la incertidumbre, con el resultado de hacerla más manifiesta, por ejemplo, en el énfasis puesto en las cosas más obvias y la reticencia con la que se anuncian las más importantes no en definitiva, la mímesis es el método resolutivo: un decir que trata de decir, no lo que es, sino aquello que otros han dicho o dirán, apoyándose en su querer decir.»

Conocí muy bien la incertidumbre que nace de la falta de autoridad en mis primeros años de trabajo. Era entonces una ingenua investigadora llena de fe en la fuerza del método científico y ansiosa por alcanzar la tranquilidad que su uso me proporcionaría para obtener resultados a la altura de mis ambiciones. Era extraordinariamente escrupulosa en el điseño y ejecución de mis experimentos, pero encontraba dificultades imprevisibles, hasta la parálisis, en el momento de validar los resultados. Pasé años recopilando datos para sostener eficazmente las hipótesis que quería demostrar. Me faltaba la práctica de la autoridad y esta carencia me impedía encontrar un punto de equilibrio entre mi deseo y lo concreto del mundo.

Salí de aquella incertidumbre al encontrar la meditación de una colega que me dijo que lo que había hecho era más que suficiente para justificar una hipótesis, autorizándome aśí a poner fin a la búsqueda de la sustentación experimental y a afrontar el juicio de la comunidad científica. Fue mi iniciación y, desde entonces, bien o mal, mi puesto ha estado asegurado.

Aquella lejana experiencia me enseñó que no basta una honesta y escrupulosa observación de las reglas del método científico, aprenđidas en los libros, para obtener la capacidad de decir lo que se sabe, sino que se necesita la mediación de las relaciones con otros investigadores, según normas precisas y dentro de estructuras e instituciones codificadas, aunque elásticas y variables, según la necesidad del momento.

El haber sido capaz de ver este hecho me ha permitido reconocer la autoridad de la ciencia, sin oscilar entre la sujección ciega y la crítica impotente. También me ha permitido comprender que el dar y recibir autoridad requiere una práctica atenta de las relaciones, porque el confín que separa la autoridad que enriquece del autoritarismo que declama puede ser muy sutil.

En efecto, puesta la atención en las relaciones que constituyen la sociedad científica, no es difícil darse cuenta de que ésta tiene una fuerte impronta masculina, que hace muy difícil la integración armoniosa de las mujeres. Esta connotación masculina viene determinada históricamente por el hecho de que la ciencia moderna se constituyó en una sociedad de abierto dominio masculino en la que, a las mujeres, se las mantenía alejadas de los estudios. Las metâforas utilizadas por los fundadores de este pensamiento son muy explícitas, tal como nos cuenta el hermoso libro de Carolyn Merchant (1991) The death of nature.
Esta parcialidad se encuentra tambiến en otros sectores de la sociedad, pero en la ciencia se oculta bajo el presupuesto de la neutralidad del objeto del conocimiento cientifico, de forma particularmente persistente. Forma parte de aquella visión que sitúa en el centro de la ciencia la relación estre sujeto y objeto de la indagación, y que requiere que, en esta relación, el sujeto entre libre de cualquier connotación particular, con actitud distante e indiferente, que es la que se define como neutra. Esta visión, abiertamente en contraste con el funcionamiento real de la emprresa científica, ejerce una gran fascinación dentro y fuera del ambiente de la investigación, y genera una disimetría en la distribución de mujeres y hombres en su seno.

Aquel sujeto universal se hace visible en la estructura masculina de la comunidad cientifica y colorea sexualmente la autoridad que alli circula. El pretendido sujeto neutro es masculino. Esto hace que la autoridad cientí. fica excluya ser mujer en el horizonte de lo que es significativo $\mathrm{y}$, por tanto, limite las posibilidades de libre expresion de las mujeres.

Una consecuencia significativa de ello es el hecho de que aún hoy, a pesar de la afluencia masiva de mujetes a las facultades científicas y a los centros de investigación, a pesar de la presencia de tantas grandes científicas y del redescubrimiınto de otras mujeres que, en el pasado, se arriesgaron a sufrir escarnio para conseguir demostrar su amor a la cienzia, sigue siendo de general aceptación que el conocimiento científico no es apto para mujeres. Así lo propagan los tests que "prueban" la menor tendencia de las chicas hacia las matemáticas, explicada de vez en cuando por razones biologicistas, otras veces por razones ambientales a por el miedo a perder admiradores si se dedican a estidios «masculinos». Consecuentemente se diseñan iniciativas para animarlas a escoger estudios científicos, dentro de una lógica de igualdad de oportunidades y acc ones positivas. En esta línea se han mantenido tesis sobre el origen social de la falta femenina en la conformación de la ciencia, del que puede ser ejemplo, un serio debate mantenido en American Journal of Physics, sobre por qué las chicas americanas no se inscriben en física y en matemáticas.

La autoridad científica, tal como se constituye actualmente, no es suficiente para que una mujer «afirme aquello que es". Tenemos necesidad de una autoridad femenina para que la autoridad que viene dada por la comunidad científica sea plena y, por tanto, comprenda y haga significativa nuestra experiencia femenina del mundo.

La práctica política de las mujeres y las reflexiones consiguientes nos muestran que, para practicar libremente el amor por el conocimiento, es necesario reconocer $\mathrm{y}$ asumir autoridad, y que la autoridad nace de las relaciones, y está, por tanto, marcada por las personas concretas con las que se entra en relación, en primer lugar por la diferencia sexual.

Cada persona debe construirse su posibilidad de palabra, teriiendo contiluamente presente lo que es y buscando la mediación adecuada a su deseo y al contexto en el que está inmersa. 
Queda clara, de esta manera, una de las causas de la dificultad en la que se encuentra quien enseña: la separación entre ciencia y enseñanza de que he hablado al principio impide aquellas relaciones de las que nace la autoridad y a través de las cuales circula la autoridad que da la seguridad necesaria para escoger, cortar e interpretar, transmitiendo a alumnas y atumnos no sólo un patrimonio de conocimiento, sino el aprendizaje necesario para una relación libre y rigurosa con ia realidad.

\section{MEDIR UNA RELACIÓN}

Ahora sabemos que es necesaria una mediación que mantenga abierta la comunicación entre enseñanza e investigación. Se trata de una necesidad que ya ha sido captada tanto por quien enseña como por quien se ocupa de la enseñanza, y este mismo congreso es una prueba elocuente. En él se han dedicado gran cantidad de trabajos, reflexiones teóricas y experiencias prácticas al funcionamiento de la escuela. La ciencia de la enseñanza es una disciplina fuertemente estructurada, rica en personas expertas y dotada de un amplio y propio patrimonio de investigación. Lo que puede parecer paradójico, aunque no lo sea, es que a pesar de ello las relaciones entre expertos y enseñantes no tienen la intensidad y la apertura necesarias para constituirse en una mediación adecuada.

La ciencia de la educación, como su nombre indica, declara compartir el estatuto actual de la ciencia y, por lo tanto, la compartimentación en disciplinas, la especialización y una idea de cientifismo que retoma la elaborada por el estudio de los fenómenos naturales, considerando científico sinónimo de exacto, repetible, intercambiable y no subjetivo.

\section{La ciencia de la enseñanza:}

- Tiene por objeto una relación entre las personas.

- Por eso tiene problemas de medida (¿quién define el metro?).

- Existe una relación entre autoridad y medida.

- Fija los criterios que han de guiar la medida.

- Garantiza el significado de la medida.

- Mide las cosas vivientes.

- Ha de contabilizar la relación entre personas y la necesaria objetividad de la medida.

Comparte, por tanto, los límites de comunicación y de eficacia, acrecentados por problemas especificos debidos ai hecho de que toma como objeto de estudio las relaciones entre personas. Como otras disciplinas que utiłizan una aproximación al conocimiento de los seres vivos, las ciencias de la educación tienen que satisfacer dos exigencias contradictorias: la de encontrar lo que es confrontable y generalizable en los objetos de su estudio y la de respetar la unicidad y la variabilidad.
Un estudio, para ser cientffico, requiere medida. La existencia de datos experimentales proporcionados por medidas bien hechas es la condición necesaria para que se pueda dar una investigación cientffica. Es común pensar en Ia medida como una relación unívoca entre un sujeto y un objeto: un sujeto que busca un orden capaz de dar sentido -comprensible para él- a un conjunto de evidencias experimentales y un objeto que se pliega dócilmentı o debe ser obligado a plegarse a las condiciones que hacen la medida significativa.

Pero una rnedida sólo tiene significado si circula por el interior de una comunidad que pueda colocarla en una tradición ce pensamiento y de investigación. Igual que la autoridad, la medida nace de las relaciones.

Existe una estrecha relación entre autoridad y medida: de hecho, la autoridad de Ia comunidad científica interviene a vezes como sostén y garantía de la actividad experimental, primero fijando los criterios a los que atenerse ps:ra obtener una medida digna de crédito $y$, luego, discutiendo las medidas efectuadas y autorizando la difusión de aquéllas que responden a los criterios definidos y aceptados.

En el transcurso de los siglos, el desarrollo de ta ciencia ha produciclo poco a poco los códigos experimentales más adecuados al estado del conocimiento y a la disponibilidad de técnicas y, actualmente, el sistema de nor-

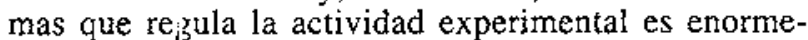
mente vasto y articulado. Sin embargo, existen algunos principios fundamentales comunes, cuyo respeto es universalmente considerado necesario para que una medida pueda definirse como tal: que sea repetible, aue su error asociado sea valorable e inferior al límite fijado por la necesaria précisión, que sea posible reconocer y controlar Ias fuentes de errores sistemáticos y que la perturbación del sistema debida a la medida sea despreciable.

Al proyectar una medida, por tanto, nos esforzamos para que la incertidumbre experimental sea lo más pequeña posible, tratando de prever y de reducir la influencia de los factores externos y de simplificar separando las diversas variables y delimitando el campo de indagación del fenómeno que se quiere estudiar.

Esta necesidad junto al uso de tecnologías muy complejas, que implican una relación muy indirecta entre quien observa y el fenómeno observado, imponen un segundo nivel de la actividad experimental, en el que, de la masa de datos obte:tidos, se hace emerger un orden que le da su significado. Éste es el momento en el que la identidad del objeto, antes subdividida y simplificada, se recompone como sintesis de las informaciones contenidas en las medidas. si se ha mantenido la coherencia entre las motivaciones de la investigación y las simples decisiones que han definido los diversos actos de la medida, esta síntesis podrá producir nuevo conocimiento.

Sin embargo, cuando las medidas se refieren a personas, muchas de las condiciones de fiabilidad de las mediciones resultan de difícil aplicación; entre ellas, Ia repetibilidad, porque un ser viviente evoluciona continuamente y porque los seres humanos no son intercambiables entre sí, 
excepto en una aproximación muy grande o reuniendo grandes muestras. Por otra parte, es muy difícil hacer de una persona viva un sistema cerrado, prescin. diendo de los factores externos. La medida tiene lugar dentro de una relación entre personas, que está siempre dotada de reciprocidad y no deja imperturbable a ninguna de las partes.

La tensión entre universal e individual que caracteriza las ciencias de las personas requiere una práctica constante y atenta de las relaciones entre expertos y objeto de estudio. Quien siga ciegamente el estándard de cientificidad elaborado para el estudio de los fenómenos naturales transforma la articulación de los dos momentos, la recogida de datos y la búsqueda del sentido, en una separación, en la cual la búsqueda del sentido es la que se pierde. Una demostración de esta pérdida son los clamorosos errores científicos, convertidos en espantosa arma de discriminación, justificados con el uso de la estadística: sirvan, como ejemplo, los tests de inteligencia.

El uso incorrecto de la estadística no deriva únicamente del hecho de que no se respetan las condiciones bajo las que han sido elaboradas las leyes estadísticas, estableciendo correlaciones arriesgadas y simplificaciones excesivas, sino también del hecho de considerar a todas las personas iguales y libres de diferencias y evolución.

\section{RECIBIR MEDIDA PARA PODER DARLA}

La capacidad para mantener unidas las relaciones y la objetividad necesaria para la medida, de forma que la inevitable articulación no se convierta en separación, es el problema central del acercamiento científico al co. nocimiento de las personas. Las soluciones posibles son probablemente más de una, diferenciadas por los diversos ámbitos experimentales.

Me interesa aquí examinar las implicaciones del caso de la enseñanza:

- No se le reconoce el aspecto de investigación.

- Necesita una valoración de la capacidad y del nivel de aprendizaje.

- La evaluación es una medida, por ello necesita autori. dad y relación.

- La cicncia de la educación no considera la relación didáctica como el centro: dedica una atención casi exclusiva a las técnicas.

- Una cientificidad que no toma en consideración el campo experimental.

Habitualmente no se reconoce el aspecto de investiga. ción que tiene la enseñanza; quienes se dedican a ella, con frecuencia, no tienen conciencia de esta situación. Sin embargo, la enseñanza, para ser efícaz, requiere una cuidadosa evaluación de la capacidad y del nivel de aprendizaje del alumnado, para que se puedan adecuar continuamente las formas y modos del proyecto didáctico.

Como toda medida, la evaluación requiere autoridad y relaciones que la hagan significativa. Pero la organización actual de la escuela hace difícil individualizar con precisión cuáles pueden ser estas relaciones. Por un lado está el estamento docente, cada persona en su clase con una discrecionalidad que parece absoluta. Por otro, están las instituciones, que, cuando no están ausentes, están interesadas $\in n$ la estandarización, la homogeneización y la intercambriabilidad. En medio, en una posición de mediación, t:stán las disciplinas específicas tales como la docimologyia, la pedagogía y otras.

El problema es que muchas veces la mediación no es adecuada. Etı primer lugar, la relación entre expertos de las ciencias de la educación y enseñantes es escasa o no existe. De hecho, el cientifismo mal entendido lleva a sobrevalorar los datos como objetivos, las estadísticas, los cuestionarios, los tests, etc., respecto a una más fatigosa confrontación en el lugar en el que se desarrolla el hecho didéctico concreto.

De esta manera se pierde lo que de verdad hace cientifico un estudio, y las investigaciones se quedan en abstracciones, incapraces de informar sobre lo que sucede, porque quedan privadas del hecho experimental que sólo quien hace el irabajo de campo, y verifica continuamente los efectos de la práctica educativa, posee, es decir, quien enseña.

Nace entonces ei sentido de una deficiencia y se recurre a posteriores nediaciones institucionales, como formadores, o técnicas, o se inventan cuestionarios cada vez más minuciosos y complicados, exigencias formales que deberán sustiluir las relaciones inexistentes, haciendo desaparecer, $t$ ajo la ficticia homogeneidad de los números y las tablasi, la diversidad en la cual está el sentido de la enseñanza. De esta forma se resta libertad y autoridad a quien enseña, sin ganar nada en cuanto a cientificidad de los resultaclos.

\section{NOTA}

* Ponencia pre:;entada al IV Congreso Internacional sobre Investigación en a Didáctica de las Ciencias y de las Matemáticas, celebrado en Bárcelona los días 13 al 16 de Septiembre de 1993. Ha sido raducida del italiano por M. Álvarez y C. Pereiro.

\section{REFERENCIA:S BIBLIOGRÁFICAS}

ALIOLI, A., 1932. Insegnare scienza: autorità e relazioni. (Rosenberg e Sellier), p. 47.

MURANO, L., 1991. L'ordine simbolico della madre. (Editori Riuniti).

MERCHANT, C., 1991. The death of nature (Harper \& Row). 